\title{
New numerical tools to study waves and instabilities of flowing plasmas
}

\author{
A.J.C. Belien ${ }^{1}$, M.A. Botchev ${ }^{2}$, J.P. Goedbloed ${ }^{4}$, B. van der Holst ${ }^{3}$, R. Keppens \\ FOM-Institute for Plasma Physics, Association Euratom-FOM \\ P.O.Box 1207, 3430 BE Nieuwegein, the Netherlands.
}

\begin{abstract}
Studying plasma waves and instabilities is an indispensable part of present thermonuclear fusion and astrophysical magnetohydrodynamics (MHD). Up till recently, spectral analysis was mostly restricted to static plasmas. However, the assumption of a static plasma is unrealistic not only for astrophysical but also for modern fusion research. Plasmas with flow have been shown to have spectra essentially different from those of static plasmas [7].

We present two new numerical tools for spectral studies of plasmas with flow. The first one, a program called FINESSE (Finite Element Solver for Stationary Equilibria), computes equilibria of non-static plasmas for a variety of fusion and astrophysical configurations (tokamaks, solar loops, solar winds, etc.).

Ideal and resistive spectra of the computed equilibria are studied with another tool, a program called PHOENIX. In PHOENIX, the large-scale generalized eigenporoblems are solved with the recently proposed iterative JacobiDavidson method [6].

Our numerical examples show how FINESSE and PHOENIX can be used to study the effect of the poloidal flows on Toroidal Alfvén Eigenmodes.
\end{abstract}

Key words: PDEs, finite elements, magnetohydrodynamics, axisymmetric equilibrium, eigenvalue problems, Jacobi-Davidson

\footnotetext{
1 Presently at: Shell International Exploration and Production

2 Presently at: Math Sciences Faculty, University of Twente, the Netherlands, e-mail: m.a.botchev@math.utwente.nl

3 Presently at: Center for Plasma Astrophysics, K.U. Leuven, Belgium

4 Corresponding author, e-mail: keppens@rijnh.nl
}

Preprint submitted to Computer Physics Communications

\section{FINESSE: Finite Element Solver for Stationary Equilibria}

The transonic equilibrium program FINESSE (Fig. 1) solves the following set of stationary ideal MHD equations:

$$
\begin{aligned}
& \nabla \cdot(\rho \mathbf{v})=0, \\
& \rho \nabla\left(\frac{1}{2} v^{2}+\frac{\gamma}{\gamma-1} \rho^{\gamma-1} S\right)-\frac{1}{\gamma-1} \rho^{\gamma} \nabla S \\
& \quad-\rho \mathbf{v} \times \omega-\mathbf{J} \times \mathbf{B}=0,
\end{aligned}
$$


FINESSE: Finite Element Solver for Stationary Equilibria

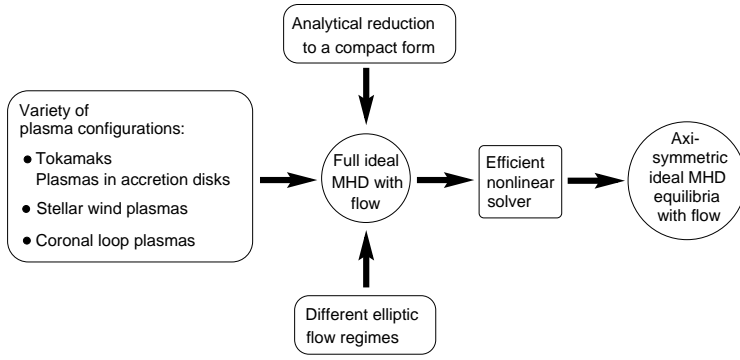

Fig. 1. Schematic representation of the FINESSE code.

$$
\begin{aligned}
& \mathbf{v} \cdot \nabla S=0, \\
& \nabla \times(\mathbf{v} \times \mathbf{B})=0, \\
& \nabla \cdot \mathbf{B}=0,
\end{aligned}
$$

where the density $\rho$, velocity $\mathbf{v}$, entropy $S$, and magnetic field $\mathbf{B}$ are the unknowns. Here $\boldsymbol{\omega} \equiv \nabla \times$ $\mathbf{v}$ is the vorticity, and $\mathbf{J} \equiv \nabla \times \mathbf{B}$ is the electric current density ( $\mu_{0}=1$ in the normalization used). The pressure $p$ is related to $S$ via $p \equiv S \rho^{\gamma}$, where $\gamma$ is the ratio of specific heats of the plasma.

Using a cylindrical coordinate system $(R, Z, \varphi)$ and axisymmetry $(\partial / \partial \varphi \equiv 0, \varphi$ is the toroidal angle), and introducing the poloidal magnetic flux function $\psi$ and the poloidal stream function $\chi$, one can show $[2,3]$ that the system $(1-5)$ can be reduced to a system of two equations in two unknowns: $\psi$ and the squared poloidal Mach number $M^{2} \equiv \rho v_{p}^{2} / B_{p}^{2}$, whereas the other unknowns can be expressed via five functions of $\psi$ that can be chosen arbitrarily. One of the equations of the new reduced system is a nonlinear PDE in $\psi$ for known $M^{2}$. The other equation is a nonlinear algebraic equation in $M^{2}$ for known $\psi$.

The reduced system is efficiently and accurately solved in FINESSE with a special iterative process [1].

\section{PHOENIX: Spectral studies of plasmas with transonic flows}

The spectral analysis of the computed equilibria is done with the help of another new code called PHOENIX (Fig. 2). PHOENIX is a successor of the known code CASTOR [4]. The fundamental differ- ence between CASTOR and PHOENIX is the ability of PHOENIX to deal with flowing plasmas. As a starting point in PHOENIX, the linearized ideal or resistive MHD equations are taken. The equations are discretized on a special, so-called straight field line coordinate grid $(\psi, \vartheta)$. Here $\vartheta$ is the poloidal angle defined by the requirement that the field lines are represented by the straight lines in the $(\varphi, \vartheta)$ plane, so that

$$
\left.(d \varphi / d \vartheta)\right|_{\text {field lines }}=J B_{\varphi} / R=q(\psi),
$$

where $J$ is the Jacobian of the $(\psi, \vartheta, \varphi)$ coordinate system, and $q$ is the so-called safety factor. The use of the $(\psi, \vartheta)$ coordinates aligned with the magnetic field is crucial for successful spectral studies. The equilibria delivered by FINESSE are transformed onto the straight field line coordinates $(\psi, \vartheta)$.

The linearized MHD equations are discretized by cubic or quadratic Hermite finite elements in the $\psi$ direction and by a Fourier spectral method in the $\vartheta$ direction. The advantage of this mixed discretization is argued in [4]. The linear perturbations are taken in the form

$$
f_{1}(s, \vartheta, \varphi, t)=e^{\lambda t} e^{i n \varphi} \sum_{m} \hat{f}_{1, m} e^{i m \vartheta}, \quad s \equiv \sqrt{\psi},
$$

where $\lambda \equiv-i \omega$ is an eigenvalue whose real part means damping or growth. The chosen form of linear perturbations is possible since the equilibria do not depend on $\varphi$. This leads to a large-scale generalized eigenvalue problem

$$
A x=\lambda B x, \quad A \neq A^{H}
$$

with complex block tridiagonal matrices $A$ and $B$, that is solved by the recently developed iterative Jacobi-Davidson method [6]. This method allows to find eigenvalues in a region of interest and the corresponding eigenvectors fast and with unprecedented accuracy. The implementation of the Jacobi-Davidson method for PHOENIX is parallelizable [5].

\section{Numerical examples}

The ideal continuous spectrum of static tokamak plasmas has Alfvén gaps where global toroidal 
PHOENIX: Waves and instabilities of plasmas with transonic flows

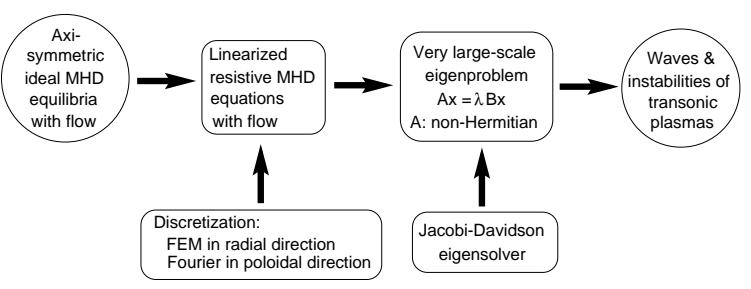

Fig. 2. Schematic representation of the PHOENIX code.

Alfvén eigenmodes (TAEs) may appear. The concept of static plasma is unrealistic for modern tokamaks where neutral beam heating and divertor pumping are used. Flow can seriously influence the global dynamics of tokamaks. As shown in [7], toroidal flow enlarges the Alfvén gaps of the ideal static MHD spectra and induces new global waves inside the gaps. These toroidal-flow-induced Alfvén eigenmodes (TFAE's) are - in contrast to the usual TAEs - in the low-frequency range and thus important for stability. Here, we consider the effects of poloidal flow on the TAEs.

We present here spectral results for a tokamak equilibrium delivered by FINESSE in the slow flow region (see [2,3]). The tokamak has a circular crosssection and inverse aspect ratio $\epsilon=0.05$.

In Fig. 3, the squared poloidal Alfvén Mach number $M^{2}$ together with the ratio $M^{2} / M_{c}^{2}$ are shown, where $M_{c}^{2} \equiv \gamma p /\left(\gamma p+B^{2}\right)$. In Fig. 3, we clearly see that $M^{2}$ is a strongly varying function of $\vartheta$ in the slow flow domain, $M_{c}^{2}<M^{2}<1$. This is characteristic for the slow flow region where the poloidal Alfvén Mach number has exceeded the critical value $M_{c}$.

A part of the ideal continuous spectrum for this equilibrium as computed by the PHOENIX code is plotted in Fig. 4. A gap in the $m=1,2$ Alfvén branches occurs at $s \approx 0.82$. The resistive spectrum at the position of this gap is shown in Fig. 5a. As can be seen in Fig. 6a, the eigenvalue A of Fig. 5a corresponds to a typical resistive wave with a rapidly oscillating eigenfunction achieving maximum amplitude around $s \approx 0.15$. On the other hand, for vanishing resistivity, a new eigenvalue (indicated by B in Fig. 5b) appears in the gap of the continuous spectrum. It is a new global Alfvén eigenmode driven by the poloidal flow with global
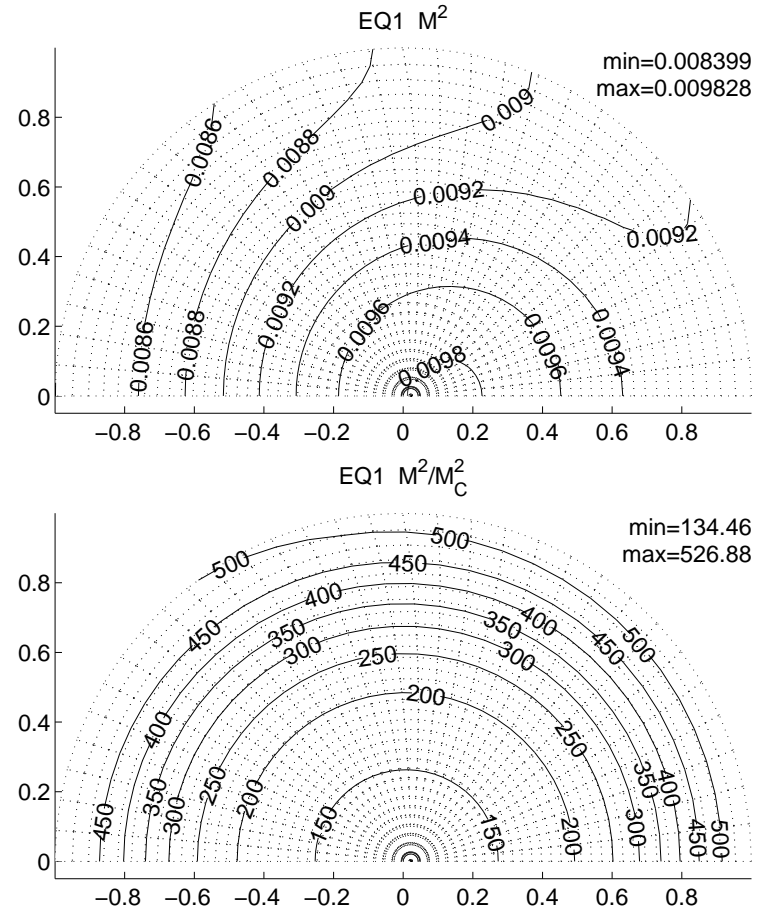

Fig. 3. Top half of a tokamak cross-section with the distribution of the squared poloidal Alfvén Mach number, $M^{2}$, and the ratio $M^{2} / M_{c}^{2}$. The plots are superposed on a straight field line coordinate grid $(\psi, \vartheta)$ shown by a dotted line.

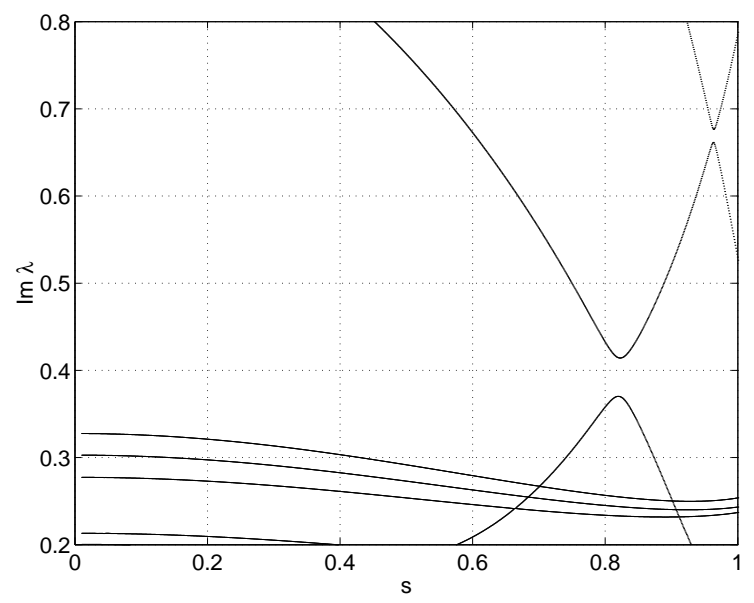

Fig. 4. Gap in the continuous spectrum of the tokamak equilibrium plotted against $s \equiv \sqrt{\psi}$. 

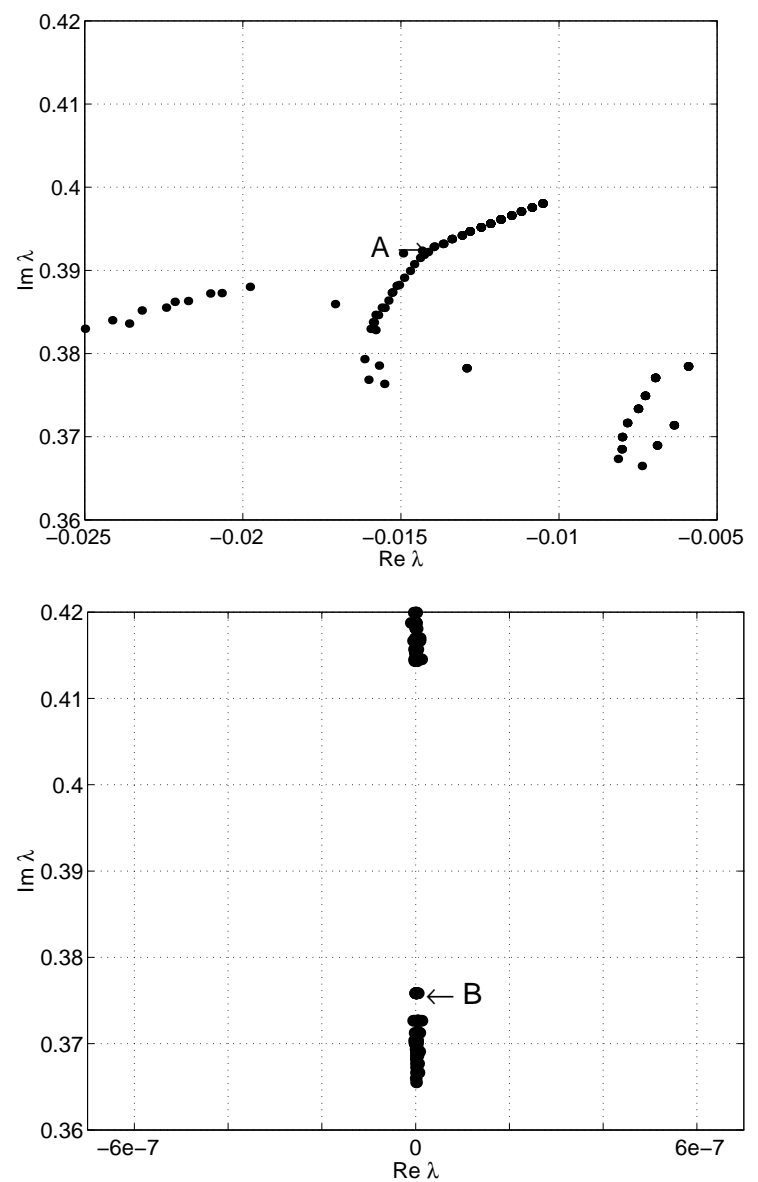

Fig. 5. Resistive spectrum in the gap for resistivity $\eta=10^{-5}$ (top) and ideal continuous spectrum with discrete eigenmodes. For the indicated eigenvalues A and B, the eigenfunctions are presented in Fig. 6 . The new ideal global mode B in the gap of the continuous spectrum is driven by transonic poloidal flows.

$m=1,2$ character (Fig. 6b).

\section{Conclusions}

We have presented two new MHD codes, FINESSE and PHOENIX, that efficiently and accurate compute plasma equilibria with flow and resistive or ideal spectra of these equilibria, respectively. The presented example demonstrates how the new codes can be used to study the influence of poloidal flow in tokamak plasmas on the toroidal
A
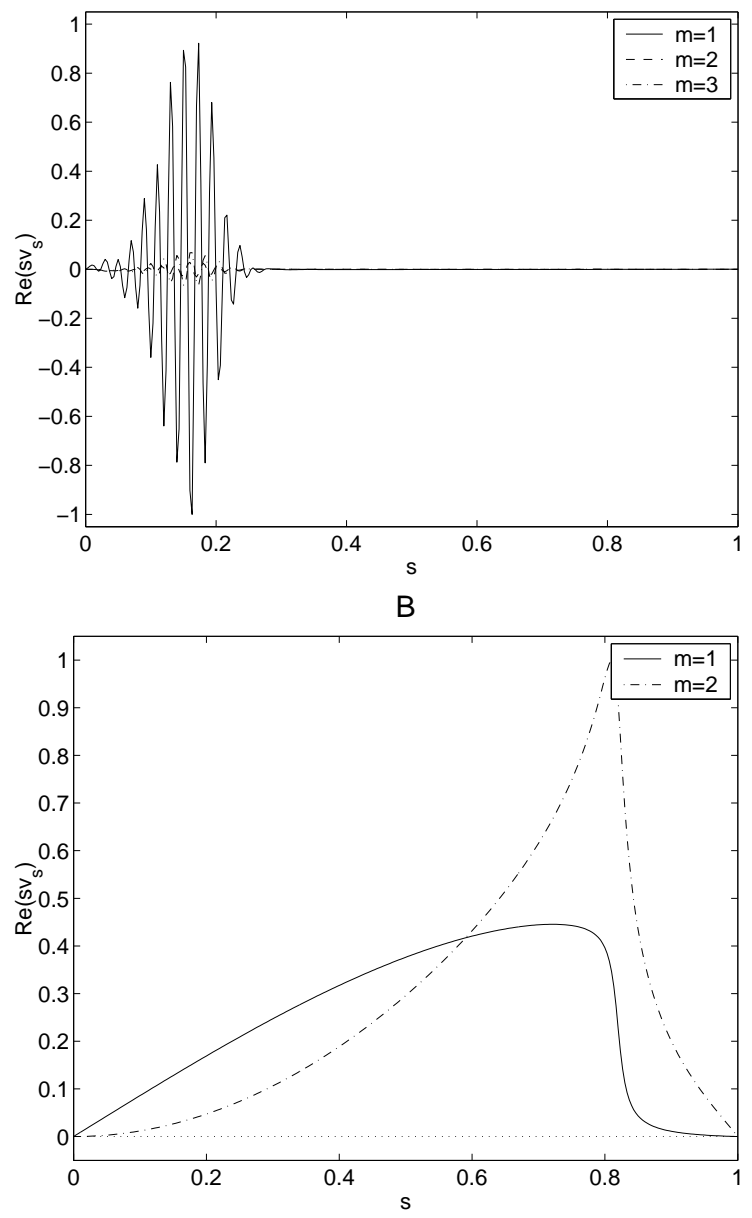

Fig. 6. Eigenfunctions of the resistive eigenvalue A (top) and the new ideal global gap mode B.

Alfvén eigenmodes (TAEs).

\section{References}

[1] Belien, A.J.C., Botchev, M.A., Goedbloed, J.P., van der Holst, B., Keppens, R., J. Comput. Phys., submitted (2001).

[2] Goedbloed, J.P., Lifschitz, A., Phys. Plasmas 4, 3544 (1997).

[3] Goedbloed, J.P., Lifschitz, A., to be published (2001).

[4] Kerner, W., Goedbloed, J.P., Huysmans, G.T.A., Poedts, S., Schwartz, E., J. Comput. Phys. 142, 271 (1998). 
[5] Nool, M., van der Ploeg, A., SIAM J. Sci. Comput. 22, 95 (2000).

[6] Sleijpen, G.L.G., van der Vorst, H.A., SIAM J. Matrix Anal. Appl. 17, 401(1996).

[7] Van der Holst, B., Belien, A.J.C., Goedbloed, J.P., Phys. Rev. Lett., 84, 2865(2000). 